\title{
Proton polarizabilities from Compton data using covariant chiral effective field theory
}

\author{
Vadim Lensky ${ }^{1,2, *}$ and Judith McGovern ${ }^{1, \dagger}$ \\ ${ }^{1}$ Theoretical Physics Group, School of Physics and Astronomy, University of Manchester, Manchester, M13 9PL, United Kingdom \\ ${ }^{2}$ Institute for Theoretical and Experimental Physics, B. Cheremushkinskaya 25, 117218 Moscow, Russia
}

(Received 16 January 2014; published 10 March 2014)

\begin{abstract}
We present a fit of the spin-independent electromagnetic polarizabilities of the proton to low-energy Compton scattering data in the framework of covariant baryon chiral effective field theory. Using the Baldin sum rule to constrain their sum, we obtain $\alpha_{E 1}=[10.6 \pm 0.25$ (stat) \pm 0.2 (Baldin) \pm 0.4 (theory) $] \times 10^{-4} \mathrm{fm}^{3}$ and $\beta_{M 1}=$ $[3.2 \mp 0.25$ (stat) \pm 0.2 (Baldin) $\mp 0.4$ (theory) $] \times 10^{-4} \mathrm{fm}^{3}$, in excellent agreement with other chiral extractions of the same quantities.
\end{abstract}

DOI: 10.1103/PhysRevC.89.032202

PACS number(s): 12.39.Fe, 13.60.Fz, 14.20.Dh

The electromagnetic polarizabilities of the proton have been a subject of investigation for many years; the earliest extractions from low-energy Compton scattering data were carried out in the 1950s, and the relevant database was greatly expanded in the 1990s. In the very-low-energy regime one can make an expansion of the cross section which deviates from the Powell one only through the inclusion of the two spinindependent polarizabilities $\alpha_{E 1}$ and $\beta_{M 1}$. However, since very few measurements have been taken below $80 \mathrm{MeV}$, almost all extractions require theoretical input to describe the evolution of the cross section with energy. Historically, dispersion relation (DR) approaches were used, with input from pion photoproduction data. A model-independent constraint can be obtained from the Baldin sum rule, most recently evaluated to give $\alpha_{E 1}+\beta_{M 1}=13.8 \pm 0.4$ in units of $10^{-4} \mathrm{fm}^{3}$ [1], so typically the parameter extracted is $\alpha_{E 1}-\beta_{M 1}$. In 2001 Olmos de León et al. published the most comprehensive data set yet, obtained with the TAPS detector at the Mainz Microtron, and in a DR framework they analyzed it together with other "modern" data to give $\alpha_{E 1}-\beta_{M 1}=10.5 \pm 0.9 \pm 0.7$ in the same units [1]. For some time this was regarded as the definitive result.

However, chiral effective field theories ( $\chi$ EFTs) can also be used to describe Compton scattering amplitudes. These are field theories in which the interactions of low-energy degrees of freedom are governed by the symmetries of QCD, and scattering amplitudes can be systematically expanded in powers of the ratio of light to heavy scales. The former are typically external particle momenta of the order of the pion mass, and the latter are governed by those particles such as the $\rho$ meson which are not included explicitly in the theory but whose effects, along with other short distance physics, are encoded in low-energy constants. At leading one-loop order in the theory with pions and nucleons these predictions are parameter free, but beyond leading order $\alpha_{E 1}$ and $\beta_{M 1}$ are free parameters which can be fit to data. The first attempt to do this was the work of Beane et al. [2,3], working in heavy baryon (HB) chiral perturbation theory. The absence of a dynamical $\Delta$ isobar restricted the fit to relatively low momentum transfer,

\footnotetext{
*lensky@itep.ru

†Judith.McGovern@manchester.ac.uk
}

and as a result the statistical errors were large. However, the inclusion of the $\Delta$ followed shortly [4-6]. Most recently, the result $\alpha_{E 1}-\beta_{M 1}=7.5 \pm 0.7 \pm 0.6$ has been obtained by McGovern et al. in Ref. [7]. Although this value is compatible with the previous chiral extractions $[2,3,6]$, the calculation was carried out to a sufficiently high order, and fit a sufficiently large set of experimental data, that the results were precise enough to demonstrate a tension with DR-based results at, roughly, the $2 \sigma$ level (combining all errors in quadrature). ${ }^{1}$

In addition to $\alpha_{E 1}$ and $\beta_{M 1}$ there are also four spin polarizabilities of the proton. One combination of these, $\gamma_{0}$, satisfies a Baldin-like sum rule and so is reasonably well known: $\gamma_{0}=-0.90 \pm 0.08$ (stat) \pm 0.11 (sys) in units of $10^{-4} \mathrm{fm}^{4}$ [9]. In looking for sources of the discrepancy in the extracted values of $\alpha_{E 1}-\beta_{M 1}$, it has been suggested that the problem is that the EFT and DR values of the spin polarizabilities are quite discrepant; since those obtained at this order in the EFT are not in good agreement with the sum-rule determination of $\gamma_{0}$, this has been used to suggest that the EFT extraction is less reliable, or at least that the errors are underestimated.

In this paper we consider the situation in a different variant of $\chi$ EFT, namely, one which does not use the heavy baryon expansion but treats the nucleon fields as Dirac spinors [10]. Compton scattering amplitudes were first calculated in this approach by Lensky and Pascalutsa in Ref. [11], using (a modification of) the extended on-mass-shell (EOMS) renormalization scheme [12]. The power-counting scheme we use is the so-called $\delta$ counting, with the small parameter $\delta \sim$ $m_{\pi} / \Delta \sim \Delta / \Lambda_{\chi} \sim 0.4$, where $\Delta=M_{\Delta}-M_{N}$ and $\Lambda_{\chi} \sim m_{\rho}$ is the chiral scale [5]. In this counting, the HB work of Ref. [7] is $O\left(e^{2} \delta^{4}\right)$ in the low-energy region. The covariant work of Ref. [11] is $O\left(e^{2} \delta^{3}\right)$, at which order $\alpha_{E 1}$ and $\beta_{M 1}$ are not free parameters but predicted. Substantial contributions to one or both come from $\pi N$ and $\pi \Delta$ loops and from $\Delta$-pole graphs, and the final values, which are the result of significant

\footnotetext{
${ }^{1}$ Because the paper is not easily available, the rather better agreement with the work of Baranov et al. [8] which, using a DR-based fit to world data, finds $\alpha_{E 1}-\beta_{M 1}=9.5 \pm 1.0 \pm 0.7$, has been less noted. In fact for "modern" pre-TAPS data alone, Baranov's result is $7.7 \pm 1.2$.
} 
TABLE I. Predictions for the spin polarizabilities in three variants of $\chi \mathrm{PT}$ and in DR, in units of $10^{-4} \mathrm{fm}^{4}$.

\begin{tabular}{lcccc}
\hline \hline & $\gamma_{E 1 E 1}$ & $\gamma_{M 1 M 1}$ & $\gamma_{E 1 M 2}$ & $\gamma_{M 1 E 2}$ \\
\hline HB $\delta^{3}[13]$ & -5.5 & 2.1 & 0.5 & 1.3 \\
HB $\delta^{4}[7]$ & -1.1 & $2.2^{\mathrm{a}}$ & -0.4 & 1.9 \\
Cov. $\delta^{3}[14]$ & -3.3 & 3.0 & 0.2 & 1.1 \\
DR [13,15] & $-3.85 \pm 0.45$ & $2.8 \pm 0.1$ & $-0.15 \pm 0.15$ & $2.0 \pm 0.1$ \\
\hline \hline
\end{tabular}

${ }^{\text {aThis value was fit in Ref. [7]; the predicted value would be }}$ $\gamma_{M 1 M 1}=6.4$.

cancellations, are $\alpha_{E 1}=10.8 \pm 0.7$ and $\beta_{M 1}=4.0 \pm 0.7$. The errors are theory only. Though no fit to data is involved, plots were presented in Ref. [11] to show that the trend of world data up to around $170 \mathrm{MeV}$ is well reproduced in this calculation. The spin polarizabilities are also predictions of the theory at this order, and interestingly they are in good agreement with the DR results, including for instance $\gamma_{0}=-0.9$; see Table I.

As yet no full calculation has been carried out in the covariant theory at $O\left(e^{2} \delta^{4}\right)$. The extra graphs required at this order are not only $\pi N$ loop graphs with insertions of second-order LECs, namely, the proton and neutron anomalous magnetic moments and the $\pi N$ scattering LECs $c_{i}$, but also photon-nucleon seagull graphs with fourth-order LECs which contribute directly to $\alpha_{E 1}$ and $\beta_{M 1}$. All of these were included in the heavy baryon calculations of Ref. [7]. There, it was shown that the contribution of the extra loop graphs was quite modest. However, the new counter-terms $\delta \alpha_{E 1}$ and $\delta \beta_{M 1}$ must be fit to Compton scattering data, and seem to be the principal new effect at this order. In view of the interest in the apparent discrepancy between DR and $\chi$ EFT extractions of $\alpha_{E 1}$ and $\beta_{M 1}$, we consider a partial $O\left(e^{2} \delta^{4}\right)$ covariant result to be of interest, and so we supplement the Lagrangian for proton fields used in [11] with the term [16,17]

$$
\begin{aligned}
\mathcal{L}_{\pi N}^{(4)}= & \pi e^{2}\left[\bar{\psi} \delta \beta_{M 1} F^{\mu \rho} F_{\mu \rho} \psi\right. \\
& \left.-\frac{2}{M_{\mathrm{N}}^{2}}\left(\delta \alpha_{E 1}+\delta \beta_{M 1}\right)\left(\partial_{\mu} \bar{\psi}\right) F^{\mu \rho} F_{\rho}^{\nu} \partial_{\nu} \psi\right] .
\end{aligned}
$$

For a review of the power counting, and of the principles underlying the application of $\chi \mathrm{EFT}$ to Compton scattering, the reader is referred to Ref. [13].

The database of experimental Compton scattering results for energies below $170 \mathrm{MeV}$, and its treatment, is the same one as was used in Refs. [7,13]; the following is only the briefest of summaries and a thorough discussion may be found in Ref. [13]. The largest single data set is from TAPS [1], for which we allow a point-to-point systematic error of $4 \%$ as advocated by Wissmann [18]; other modern data are from [1922], and a number of older experiments also contribute some points. Normalization uncertainties are incorporated into the $\chi^{2}$ function in the usual way. As in Refs. [7,13], for our final result we use the data of Hallin et al. [21] below $150 \mathrm{MeV}$ only.

We do not fit to any higher-energy data but check by eye that the agreement continues to be good at higher energies (at least as far as the deficiencies of the data sets allow such a judgment). We take the $\gamma N \Delta$ coupling constants obtained in

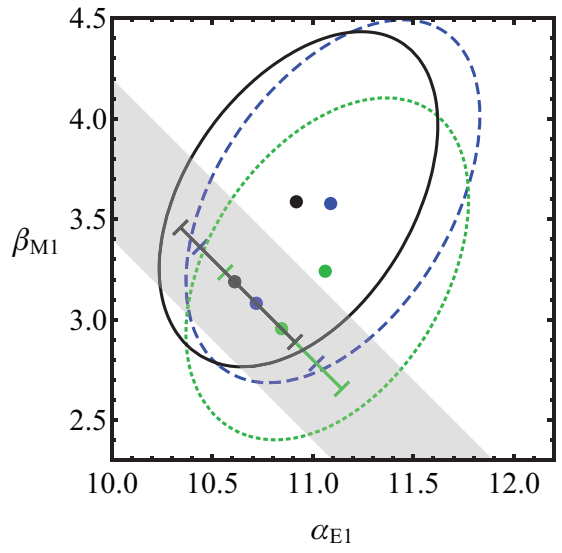

FIG. 1. (Color online) $1 \sigma$ contours for the one-parameter (Baldin-constrained) and two-parameter (unconstrained) fits, for three variants of the data set: the solid (black) line is our final result, while the dashed (blue) lines exclude all Hallin data, and the dotted (green) line uses an upper cutoff of $150 \mathrm{MeV}$. These are sets (III), (II), and (I), respectively, of Ref. [7].

a fit to photoproduction data: $g_{M}=2.97$ and $g_{E}=-1.0$ [23]. Other parameters are given in Ref. [7]. We present results both with and without imposing the Baldin sum rule $\alpha_{E 1}+\beta_{M 1}=$ $13.8 \pm 0.4[1]$ as follows:

$$
\begin{aligned}
& \alpha_{E 1}=10.9 \pm 0.45 \text { (stat) } \pm 0.4 \text { (theory), } \\
& \beta_{M 1}=3.6 \pm 0.55 \text { (stat) } \pm 0.4 \text { (theory), }
\end{aligned}
$$

with $\chi^{2}$ of 111.8 for 135 degrees of freedom (d.o.f.). For the Baldin-constrained fit we obtain $\alpha_{E 1}-\beta_{M 1}=7.4 \pm$ 0.5 (stat) \pm 0.4 (theory), giving

$$
\begin{aligned}
& \alpha_{E 1}=10.6 \pm 0.25 \text { (stat) } \pm 0.2 \text { (Baldin) } \pm 0.4 \text { (theory), } \\
& \beta_{M 1}=3.2 \mp 0.25 \text { (stat) } \mp 0.2 \text { (Baldin) } \pm 0.4 \text { (theory), }
\end{aligned}
$$

with $\chi^{2}$ of 112.5 for 136 d.o.f. The theory errors have been conservatively calculated, based on the shift of $\alpha_{E 1}-\beta_{M 1}$ from $e^{2} \delta^{3}$ to partial $e^{2} \delta^{4}$, multiplied by the parameter $\delta \sim 0.4$.

As can be seen from Fig. 1 (which also demonstrates the sensitivity to variations of the choice of database), these results are completely consistent with one another. Furthermore they are consistent with the corresponding results in the heavy baryon extraction [7], and in fact the central values of the Baldin-constrained fit are essentially equal to those of that work, namely, $\alpha_{E 1}=10.65 \pm 0.35$ (stat), $\beta_{M 1}=3.2 \mp$ 0.35 (stat). (The statistical error is higher than in the current work because an extra parameter, $\gamma_{M 1 M 1}$, was fitted in Ref. [7].) It is also interesting to recall that partial and full $O\left(e^{2} \delta^{4}\right) \mathrm{HB}$ extractions of $\alpha_{E 1}-\beta_{M 1}$ agree very well [7].

In Fig. 2 we show the fit along with a selection of data. The low-energy fit is excellent, and below the photoproduction threshold the predictions of covariant and heavy-baryon $\chi \mathrm{PT}$ are largely indistinguishable. (The similarity of the covariant and $\mathrm{HB}$ predictions for the unpolarized cross sections in this region was already noted in Ref. [24].) The values of $\alpha_{E 1}$ and $\beta_{M 1}$ in all three cases are extremely close, but the spin polarizabilities are quite disparate. The HB $O\left(e^{2} \delta^{3}\right)$ curves give a stronger cusp than the covariant version, but 

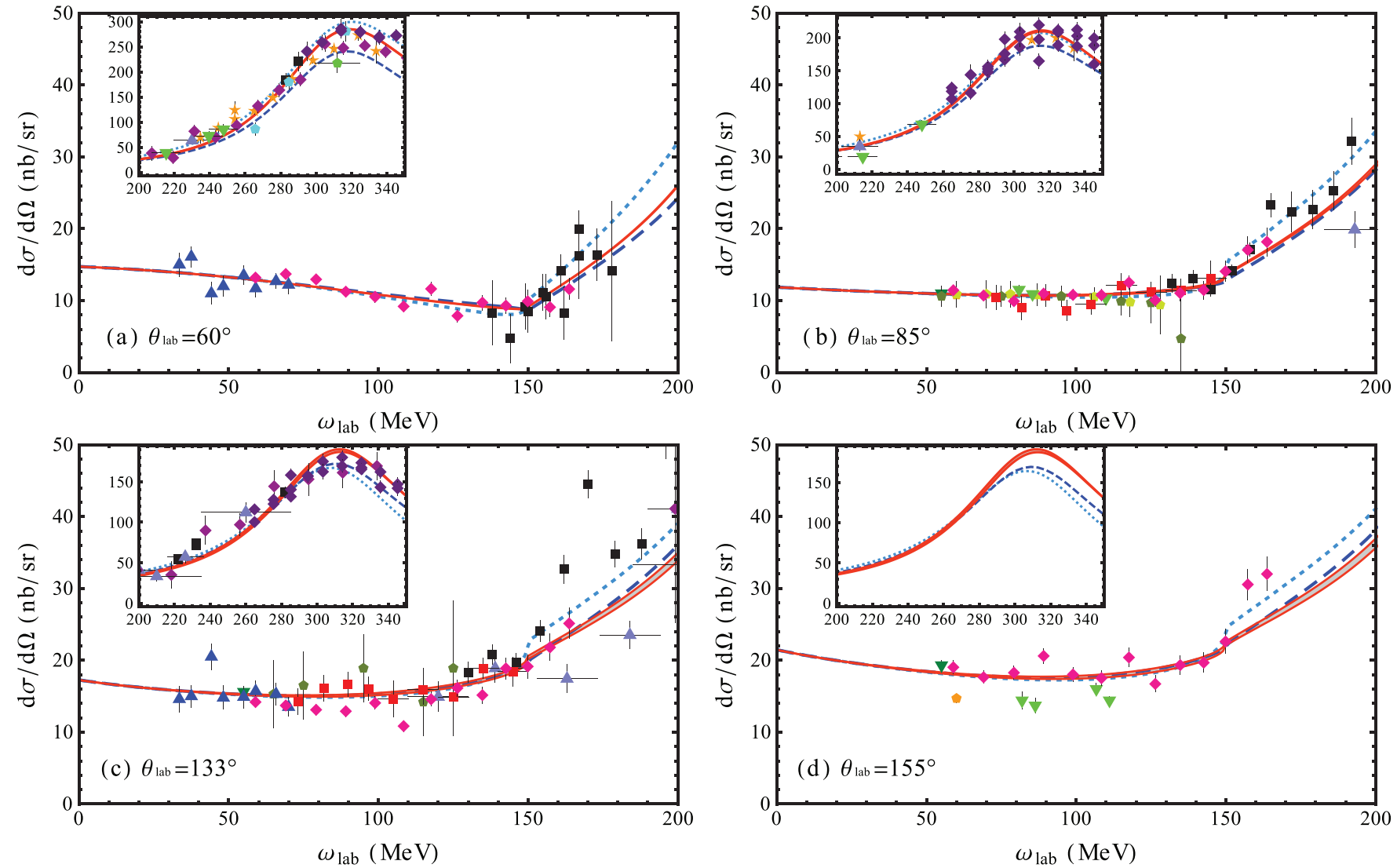

FIG. 2. (Color online) Predictions for the cross section in $\mathrm{nb} / \mathrm{sr}$ as a function of incoming photon energy in MeV (both in the laboratory frame), using the values from the Baldin-constrained fit $\alpha_{E 1}=10.6$ and $\beta_{M 1}=3.2$, and with the band showing statistical errors only (solid line, red). For comparison the results of HB $\chi \mathrm{PT}$ are shown: $O\left(e^{2} \delta^{3}\right)$ [13] (dotted, light blue) and $O\left(e^{2} \delta^{4}\right)$ [7] (dashed, dark blue). Data points are shown without floating normalization, and including those points which were excluded from the fit. The key is given in Table 3.1 of Ref. [13]; in particular purple diamonds are Mainz data [1,25]; red squares, MacGibbon [22]; black squares, Hallin [21]; and blue triangles, Federspiel [19]. The insets show the extended range beyond $\omega_{\text {lab }}=200 \mathrm{MeV}$.

the HB $O\left(e^{2} \delta^{4}\right)$ is in very good agreement with the covariant calculation up to $200 \mathrm{MeV}$ and beyond. Though only lowenergy data are used in the fit, the good agreement with the Mainz data [25] continues into the resonance region, though at most angles the $\Delta$ peak is somewhat too high. It should be noted though that in this region the power counting changes and the EFT calculation is only leading order. Moreover the HB fits varied the $\gamma N \Delta$ coupling constant $g_{M}$ whereas in the present, covariant fit we have used the value obtained in the covariant theory from photoproduction [23], which is around $10 \%$ higher. The leading dependence of the height of the peak on the coupling constant is $g_{M}^{4}$, and a better fit in the resonance region could be obtained by allowing a modest variation of this parameter with negligible effect on the low-energy fit.

In summary, we have shown that in a fit to low-energy Compton scattering data, very similar results are obtained for the electromagnetic polarizabilities of the proton, $\alpha_{E 1}$ and $\beta_{M 1}$, whether the covariant or heavy baryon versions of chiral effective field theory are used. In particular if the Baldin sum-rule constraint is applied, the extracted values of $\alpha_{E 1}-\beta_{M 1}$ are essentially identical. This result is unexpected because some other predictions of the two versions, notably the spin polarizabilities, are apparently not in good agreement. However, these are not the dominant drivers of the energy evolution at photon energies comparable to $m_{\pi}$, and the two versions of the theory make very similar predictions for the overall cross section. It should be noted that this energy dependence, including the cusp at photoproduction threshold generated by chiral loops, is highly nontrivial. The excellent fit to data with only one free parameter demonstrates the predictive power of $\chi \mathrm{EFT}$.

It is still to be tested whether the tension between the $\chi \mathrm{EFT}$ extraction and the widely accepted dispersion-relation-based one of Ref. [1] is due to the larger and more carefully handled data set used in the chiral extractions, or to some other feature of the predictions of the two theories. More unpolarized data, particularly at energies around $m_{\pi}$ at backward angles, might be needed to resolve the issue. To further explore spin polarizabilities, though, it is clear that polarized scattering measurements will be required.

We are grateful to Daniel Phillips and Harald Grießhammer for discussions regarding aspects of these calculations, and 
to Mike Birse and Vladimir Pascalutsa for useful comments on the manuscript. This work has been supported in part by
U.K. Science and Technology Facilities Council Grants No. ST/F012047/1 and No. ST/J000159/1.
[1] V. Olmos de León et al., Eur. Phys. J. A 10, 207 (2001).

[2] S. R. Beane, M. Malheiro, J. A. McGovern, D. R. Phillips, U. van Kolck, Phys. Lett. B 567, 200 (2003); 607, 320 (2005).

[3] S. R. Beane, M. Malheiro, J. A. McGovern, D. R. Phillips, and U. van Kolck, Nucl. Phys. A 747, 311 (2005).

[4] T. R. Hemmert, B. R. Holstein, and J. Kambor, Phys. Rev. D 55, 5598 (1997).

[5] V. Pascalutsa and D. R. Phillips, Phys. Rev. C 67, 055202 (2003).

[6] R. P. Hildebrandt, H. W. Grießhammer, T. R. Hemmert, and B. Pasquini, Eur. Phys. J. A 20, 293 (2004).

[7] J. A. McGovern, D. R. Phillips, and H. W. Grießhammer, Eur. Phys. J. A 49, 12 (2013).

[8] P. S. Baranov, A. I. L'vov, V. A. Petrun'kin, and L. N. Shtarkov, Phys. Part. Nucl. 32, 376 (2001).

[9] B. Pasquini, P. Pedroni, and D. Drechsel, Phys. Lett. B 687, 160 (2010).

[10] J. Gasser, M. E. Sainio, and A. Svarc, Nucl. Phys. B 307, 779 (1988).

[11] V. Lensky and V. Pascalutsa, Eur. Phys. J. C 65, 195 (2010).

[12] T. Fuchs, J. Gegelia, G. Japaridze, and S. Scherer, Phys. Rev. D 68, 056005 (2003).
[13] H. W. Grießhammer, J. A. McGovern, D. R. Phillips, and G. Feldman, Prog. Part. Nucl. Phys. 67, 841 (2012).

[14] V. Lensky, V. Pascalutsa, and J. McGovern (unpublished).

[15] D. Drechsel, B. Pasquini, and M. Vanderhaeghen, Phys. Rep. 378, 99 (2003).

[16] N. Fettes, U.-G. Meißner, M. Mojžǐš, and S. Steininger, Ann. Phys. (NY) 283, 273 (2000); 288, 249 (2001).

[17] N. Krupina and V. Pascalutsa, Phys. Rev. Lett. 110, 262001 (2013).

[18] F. Wissmann, Compton Scattering: Investigating the Structure of the Nucleon with Real Photons, Springer Tracts in Modern Physics, Vol. 200 (Springer, Berlin, 2004).

[19] F. J. Federspiel et al., Phys. Rev. Lett. 67, 1511 (1991).

[20] A. Zieger et al., Phys. Lett. B 278, 34 (1992).

[21] E. L. Hallin et al., Phys. Rev. C 48, 1497 (1993).

[22] B. E. MacGibbon et al., Phys. Rev. C 52, 2097 (1995).

[23] V. Pascalutsa and M. Vanderhaeghen, Phys. Rev. D 73, 034003 (2006).

[24] V. Lensky, J. A. McGovern, D. R. Phillips, and V. Pascalutsa, Phys. Rev. C 86, 048201 (2012).

[25] S. Wolf, V. Lisin, R. Kondratiev, A. M. Massone, G. Galler, J. Ahrens, H. J. Arends, R. Beck et al., Eur. Phys. J. A 12, 231 (2001). 